\title{
2- 上循环交叉积
}

\section{赵建伟}

中国计量学院理学院数学系, 杭州 310018

E-mail: zhaojw@amss.ac.cn

收稿日期: 2010-09-29; 接受日期: 2011-02-01

国家自然科学基金 (批准号: 10926118) 和浙江省自然科学基金 (批准号: Y6110117) 资助项目

摘要 利用 von Neumann 代数 $\mathcal{M}$ 和带有正规的 $2-$ 上循环 $\mu$ 的离散群 $G$, 定义了 2- 上循环交叉积, 推广了经典的离散交叉积, 并证明了 2- 上循环交叉积具有结合律.

关键词 von Neumann 代数 2-上循环交叉积 结合律

MSC (2000) 主题分类 $46 \mathrm{~L} 10$

\section{1 引言}

令 $\mathcal{H}$ 表示可分的 Hilbert 空间, $\mathcal{B}(\mathcal{H})$ 表示作用在 $\mathcal{H}$ 上的有界线性算子之集. von Neumann 代数 是 $\mathcal{B}(\mathcal{H})$ 中包含单位元的弱算子闭的自伴 *- 子代数. 当 von Neumann 代数 $\mathcal{M}$ 的中心 $\mathcal{C}$ 是平凡的, 即 $\mathcal{M} \cap \mathcal{M}^{\prime}=\mathbb{C} I$ 时, $\mathcal{M}$ 是因子. von Neumann 和 Murray 把因子分为 $I 、 I I$ 和 $I I I$ 型, 并且分别给 出了构造它们的方法.

利用可数离散群 $G$ 的左正则表示构造群 von Neumann 代数 $\mathcal{L}_{G}$ 是 von Neumann 代数的主要构 造方法之一. 具体的构造方法如下:

令

$$
l^{2}(G)=\left\{\xi:\left.G \rightarrow \mathbb{C}\left|\sum_{g \in G}\right| \xi(g)\right|^{2}<+\infty\right\}
$$

内积为

$$
\langle\xi, \eta\rangle=\sum_{g \in G} \xi(g) \overline{\eta(g)},
$$

则 $l^{2}(G)$ 是 Hilbert 空间. $\forall g \in G$, 令 $L(g)$ 表示如下定义的西算子,

$$
L(g)(\xi)(h)=\xi\left(g^{-1} h\right), \quad \forall \xi \in l^{2}(G), h \in G .
$$

则映射 $g \mapsto L(g)$ 是群 $G$ 在 Hilbert 空间 $l^{2}(G)$ 上的西表示, 称为群 $G$ 的左正则表示. 由西算子之 集 $\{L(g) \mid g \in G\}$ 所生成的 von Neumann 代数 $\mathcal{L}_{G}$ 称为群 von Neumann 代数 ${ }^{[1]}$. 上述构造群 von Neumann 代数的方法是 von Neumann 代数理论的重要部分, 它不仅为算子代数理论提供了许多具体 的 $I I_{1}$ 型因子的例子, 而且吸引了许多算子代数工作者对群 von Neumann 代数同构、代数不变量和 代数性质等问题的研究 ${ }^{[2-6]}$. 
Connes 和 Jones 在文献 [7] 中给出了 2- 上循环群 von Neumann 代数, 它是群 von Neumann 代数 的推广. 具体的构造方法如下:

令 $G$ 是离散群, 其单位元为 $e, \mu \in H^{2}(G, \mathbb{T})$ 是群 $G$ 上的 2- 上循环, 即 $\mu: G \times G \rightarrow \mathbb{T}$ 满足

$$
\mu(g, h) \mu(g h, k)=\mu(h, k) \mu(g, h k), \quad \forall g, h, k \in G,
$$

其中 $\mathbb{T}$ 是复平面中的单位圆. $\forall g \in G$, 令 $L_{\mu}(g)$ 表示如下定义的 Hilbert 空间 $l^{2}(G)$ 上的西算子,

$$
L_{\mu}(g)(\xi)(h)=\mu\left(g, g^{-1} h\right) \xi\left(g^{-1} h\right), \quad \forall \xi \in l^{2}(G), h \in G .
$$

则映射 $g \mapsto L_{\mu}(g)$ 称为群 $G$ 关于 $\mu$ 在 Hilbert 空间 $l^{2}(G)$ 上的投影左正则表示. 由西算子之集 $\left\{L_{\mu}(g) \mid g \in G\right\}$ 所生成的 von Neumann 代数 $\mathcal{L}_{\mu}(G)$ 称为 2 - 上循环群 von Neumann 代数 ${ }^{[7]}$. 当 $\mu \equiv 1$ 时, 2- 上循环群 von Neumann 代数 $\mathcal{L}_{\mu}(G)$ 就是群 von Neumann 代数 $\mathcal{L}(G)$. Nicoara 等在文献 [8] 中 研究了单参数 2- 上循环群 von Neumann 代数 $\mathcal{L}_{\mu_{\alpha}}\left(\mathbb{Z}^{2} \rtimes \Gamma\right)$ 的同构问题.

利用可测空间和上面的作用群构造离散交叉积是构造 von Neumann 代数的另一个重要的方法, 它是 Turumaru ${ }^{[9]}$ 以及 Nakamura 和 Takeda ${ }^{[10]}$ 定义的交叉积的特殊情形. 由于抽象的交叉积同构于 某一实现的交叉积, 所以这里我们仅回顾实现的交叉积的定义 ${ }^{[11]}$. 设 $\mathcal{M}$ 是作用在 Hilbert 空间 $\mathcal{H}$ 上 的 von Neumann 代数, $G$ 是离散群, $\alpha: g \mapsto \alpha(g)$ 是群 $G$ 在 $\mathcal{M}$ 上的自同构表示, 且由群 $G$ 在 $\mathcal{H}$ 上 的西表示 $g \mapsto U(g)$ 实现, 则 $\mathcal{M}$ 和 $\alpha$ 的 (实现) 交叉积是作用在 $\mathcal{H} \otimes l^{2}(G)$ 上的由下列算子

$$
\Phi(A)=A \otimes I, \quad V(g)=U(g) \otimes L(g), \quad A \in \mathcal{M}, g \in G,
$$

所生成的 von Neumann 代数 $\mathcal{M} \rtimes_{\alpha} G$.

吴文明和袁魏在文献 [12] 中研究了离散交叉积的结合律. 本文利用 von Neumann 代数和带有正 规的 2- 上循环的离散群 $G$ 构造 2- 上循环交叉积. 当 $\mu$ 在 $G \times G$ 上取值为 1 时, 2- 上循环离散交叉 积就是经典的离散交叉积. 进一步, 本文研究了 2- 上循环交叉积的结合律, 从而推广文献 [12] 中的定 理 3.3 .

\section{$2 \quad 2-$ 上循环交叉积的定义}

设 $G$ 是离散群, 其单位元为 $e$. 如果 $G$ 上的 2 - 上循环 $\mu$ 满足

$$
\mu(g, e)=\mu(e, g)=1, \quad \forall g \in G,
$$

则 $\mu$ 称为正规的. 在 Hilbert 空间 $l^{2}(G)$ 中, 定义 $\chi_{g}(h)=\delta_{g, h}$, 则 $\left\{\chi_{g} \mid g \in G\right\}$ 为 $l^{2}(G)$ 的正规正交 基. 令 $E_{g}$ 表示 Hilbert 空间 $l^{2}(G)$ 到 $\chi_{g}$ 所生成的一维子空间上的投影. 现在, 我们给出 (抽象) 2- 上 循环交叉积的定义.

定义 2.1 设 $\mathcal{M}$ 是作用在 Hilbert 空间 $\mathcal{H}$ 上的 von Neumann 代数, $G$ 是离散群, $\mu \in H^{2}(G, \mathbb{T})$ 是群 $G$ 上的正规 2- 上循环, $\alpha: g \mapsto \alpha(g)$ 是群 $G$ 在 $\mathcal{M}$ 上的自同构表示, 则 $\mathcal{M}$ 和 $\alpha$ 的 (抽象) 2- 上 循环交叉积是作用在 $\mathcal{H} \otimes l^{2}(G)$ 上的由下列算子

$$
\Phi(A)=\sum_{g \in G} \alpha(g)^{-1}(A) \otimes E_{g}, \quad \Psi(g)=I \otimes L_{\mu}(g), \quad A \in \mathcal{M}, g \in G,
$$

所生成的 von Neumann 代数 $\mathcal{M} \rtimes_{\alpha}^{\mu} G$. 
$\forall g, h \in G$, 取 $\mu(g, h)=1$, 则 $\mu$ 是 $G$ 上的正规 2- 上循环且 $L(g)=L_{\mu}(g)$. 因此, 我们得到如下 结论:

注 2.2 离散交叉积是 2- 上循环交叉积的特殊情形.

类似于 Kadison 和 Ringrose 在著作 [11] 中关于离散交叉积的研究, 本文将研究 (实现) 2- 上循环 交叉积. 为此, 我们先建立以下性质 2.3 .

性质 2.3 设 $\mathcal{M}$ 是作用在 Hilbert 空间 $\mathcal{H}$ 上的 von Neumann 代数, $G$ 是离散群, $\mu \in H^{2}(G, \mathbb{T})$ 是群 $G$ 上的正规 2- 上循环, $\alpha: g \mapsto \alpha(g)$ 是群 $G$ 在 $\mathcal{M}$ 上的自同构表示.

（i）如果 $\theta$ 是从 von Neumann 代数 $\mathcal{M}$ 到 $\mathcal{N}$ 上的 $*$ - 同构, $\beta$ 是群 $G$ 在 $\mathcal{N}$ 上的自同构表示 $g \mapsto \beta(g)=\theta \alpha(g) \theta^{-1}$, 则 $\mathcal{M} \rtimes_{\alpha}^{\mu} G *$ 同构于 $\mathcal{N} \rtimes_{\beta}^{\mu} G$.

$(\dot{2})$ 存在从 von Neumann 代数 $\mathcal{M}$ 到 $\mathcal{N}$ 上的 $*$ - 同构 $\theta$, 使得群 $G$ 在 $\mathcal{N}$ 上的自同构表示 $g \mapsto$ $\theta \alpha(g) \theta^{-1}$ 可以被西实现.

证明 (i) 令 $\pi=\theta \otimes I$, 其中 $I$ 是作用在 $l^{2}(G)$ 上的恒等算子, 则 $\pi$ 是从 von Neumann 代数 $\mathcal{M} \bar{\otimes} \mathcal{B}\left(l^{2}(G)\right)$ 到 von Neumann 代数 $\mathcal{N} \bar{\otimes} \mathcal{B}\left(l^{2}(G)\right)$ 上的 $*$ - 同构. 因为 $\pi\left(\mathcal{M} \rtimes_{\alpha}^{\mu} G\right)$ 是由算子 $\pi(\Phi(A))(A \in$ $\mathcal{M})$ 和 $\pi(\Psi(g))(g \in G)$ 所生成的 von Neumann 代数, 而

$$
\pi(\Phi(A))=\sum_{g \in G} \beta(g)^{-1}(\theta(A)) \otimes E_{g}, \quad \pi(\Psi(g))=I \otimes L_{\mu}(g),
$$

所以 $\mathcal{M} \rtimes_{\alpha}^{\mu} G *$ - 同构于 $\mathcal{N} \rtimes_{\beta}^{\mu} G$.

$(\dot{2})$ 令 $\theta: \mathcal{M} \rightarrow \Phi(\mathcal{M})$ 定义为 $\theta(A)=\Phi(A)=\sum_{g \in G} \alpha(g)^{-1}(A) \otimes E_{g}$, 则 $\theta$ 是 $*$ - 同构且 $\beta: g \mapsto$ $\beta(g)=\theta \alpha(g) \theta^{-1}$ 是群 $G$ 在 $\Phi(\mathcal{M})$ 上的自同构表示. 定义 $\mathcal{H} \otimes l^{2}(G)$ 上的算子 $U(g)$ 为

$$
U(g)\left(\sum_{h \in G} \xi(h) \otimes \chi_{h}\right)=\sum_{h \in G} \xi(h) \otimes \chi_{g h},
$$

则容易验证 $U(g)$ 是作用在 $\mathcal{H} \otimes l^{2}(G)$ 上的酉算子. 此时, $U: g \mapsto U(g)$ 是群 $G$ 在 $\mathcal{H} \otimes l^{2}(G)$ 上的西 表示. 由于

$$
U(g) \Phi(A) U(g)^{*}=\beta(g)(\Phi(A)), \quad \forall A \in \mathcal{M}, \forall g \in G,
$$

所以 $\mathcal{M} \rtimes_{\alpha}^{\mu} G *$ 同构于 $\Phi(\mathcal{M}) \rtimes_{\beta}^{\mu} G$, 其中西表示 $U$ 实现自同构表示 $\beta$.

设群 $G$ 在 von Neumann 代数 $\mathcal{M}$ 上的自同构表示 $\alpha$ 能够由西表示 $U: g \mapsto U(g)$ 在 $\mathcal{H}$ 上实现. 令

$$
W=\sum_{g \in G} U(g) \otimes E_{g},
$$

则 $W$ 是作用在 Hilbert 空间 $\mathcal{H} \otimes l^{2}(G)$ 上的西算子. 因为

$$
\begin{aligned}
W \Phi & (A) W^{*}\left(\sum_{g \in G} \xi(g) \otimes \chi_{g}\right)=W \Phi(A)\left(\sum_{g \in G} U\left(g^{-1}\right)(\xi(g)) \otimes \chi_{g}\right) \\
& =W\left(\sum_{g \in G} \alpha(g)^{-1}(A) \otimes E_{g}\right)\left(\sum_{h \in G} U\left(h^{-1}\right)(\xi(h)) \otimes \chi_{h}\right) \\
& =W\left(\sum_{g \in G} \sum_{h \in G} \alpha(g)^{-1}(A)\left(U\left(h^{-1}\right)(\xi(h))\right) \otimes E_{g}\left(\chi_{h}\right)\right) \\
& =W\left(\sum_{g \in G} U\left(g^{-1}\right) A(\xi(g)) \otimes \chi_{g}\right)=\sum_{g \in G} A \xi(g) \otimes \chi_{g}
\end{aligned}
$$




$$
=\left(\sum_{g \in G} A \otimes E_{g}\right)\left(\sum_{h \in G} \xi(h) \otimes \chi_{h}\right)=(A \otimes I)\left(\sum_{g \in G} \xi(g) \otimes \chi_{g}\right)
$$

所以

$$
W \Phi(A) W^{*}=A \otimes I
$$

又因

$$
\begin{aligned}
W \Psi(g) W^{*}\left(\sum_{h \in G} \xi(h) \otimes \chi_{h}\right)=W \Psi(g)\left(\sum_{h \in G} U\left(h^{-1}\right)(\xi(h)) \otimes \chi_{h}\right) \\
=W\left(\sum_{h \in G} U\left(h^{-1}\right)(\xi(h)) \otimes L_{\mu}(g)\left(\chi_{h}\right)\right) \\
=W\left(\sum_{h \in G} U\left(h^{-1}\right)(\xi(h)) \otimes \mu(g, h)\left(\chi_{g h}\right)\right) \\
=W\left(\sum_{h \in G} \mu\left(g, g^{-1} h\right) U\left(h^{-1} g\right)\left(\xi\left(g^{-1} h\right)\right) \otimes \chi_{h}\right) \\
=\sum_{h \in G} \mu\left(g, g^{-1} h\right) U(g)\left(\xi\left(g^{-1} h\right)\right) \otimes \chi_{h} \\
=\left(U(g) \otimes L_{\mu}(g)\right)\left(\sum_{h \in G} \xi(h) \otimes \chi_{h}\right),
\end{aligned}
$$

所以

$$
W \Psi(g) W^{*}=U(g) \otimes L_{\mu}(g) .
$$

因此, $W\left(\mathcal{M} \rtimes_{\alpha}^{\mu} G\right) W^{*}$ 是由 $\left\{A \otimes I, U(g) \otimes L_{\mu}(g) \mid A \in \mathcal{M}, g \in G\right\}$ 所生成的 von Neumann 代数. 由于 $W\left(\mathcal{M} \rtimes_{\alpha}^{\mu} G\right) W^{*}$ 西等价于 $\mathcal{M} \rtimes_{\alpha}^{\mu} G$, 所以建立并研究 (实现) 2- 上循环交叉积是合理的.

定义 2.4 设 $\mathcal{M}$ 是作用在 Hilbert 空间 $\mathcal{H}$ 上的 von Neumann 代数, $G$ 是离散群, $\mu \in H^{2}(G, \mathbb{T})$ 是群 $G$ 上的正规 2- 上循环, $\alpha: g \mapsto \alpha(g)$ 是群 $G$ 在 $\mathcal{M}$ 上的自同构表示, 且由西表示 $g \mapsto U(g)$ 在 $\mathcal{H}$ 上实现, 则 $\mathcal{M}$ 和 $\alpha$ 的 (实现) 2- 上循环交叉积是作用在 $\mathcal{H} \otimes l^{2}(G)$ 上的由下列算子

$$
\varphi(A)=A \otimes I, \quad \psi(g)=U(g) \otimes L_{\mu}(g), \quad A \in \mathcal{M}, g \in G,
$$

所生成的 von Neumann 代数 $\mathcal{M} \rtimes_{\alpha}^{\mu} G$.

$\forall g, h \in G$, 因为

$$
\begin{aligned}
L_{\mu}(g) L_{\mu}(h)(\xi)(k) & =\mu\left(g, g^{-1} k\right)\left(L_{\mu}(h)(\xi)\right)\left(g^{-1} k\right)=\mu\left(g, g^{-1} k\right) \mu\left(h, h^{-1} g^{-1} k\right) \xi\left(h^{-1} g^{-1} k\right) \\
& =\mu\left(g h,(g h)^{-1} k\right) \overline{\mu\left(g h,(g h)^{-1} k\right)} \mu\left(g, g^{-1} k\right) \mu\left(h, h^{-1} g^{-1} k\right) \xi\left(h^{-1} g^{-1} k\right) \\
& =\mu\left(g, g^{-1} k\right) \mu\left(h, h^{-1} g^{-1} k \overline{\mu\left(g h,(g h)^{-1} k\right)} L_{\mu}(g h)(\xi)(k)\right. \\
& =\mu\left(g, g^{-1} k\right) \mu\left(h, h^{-1} g^{-1} k\right) \mu(g, h) \overline{\mu\left(h, h^{-1} g^{-1} k\right) \mu\left(g, g^{-1} k\right)} L_{\mu}(g h)(\xi)(k) \\
& =\mu(g, h) L_{\mu}(g h)(\xi)(k) \quad\left(\forall \xi \in l^{2}(G), k \in G\right),
\end{aligned}
$$

所以

$$
L_{\mu}(g) L_{\mu}(h)=\mu(g, h) L_{\mu}(g h) .
$$

从而集合 $\left\{A U(g) \otimes L_{\mu}(g) \mid A \in \mathcal{M}, g \in G\right\}$ 的线性扩张 $\mathcal{R}_{0}$ 在 (实现) 2- 上循环交叉积 $\mathcal{M} \rtimes_{\alpha}^{\mu} G$ 中是 稠密的. 


\section{$3 \quad 2-$ 上循环交叉积的结合律}

令 $G \rtimes_{\sigma} K$ 是离散群 $G$ 和 $K$ 的半直积, 其中群 $G$ 和 $K$ 的单位元分别为 $e_{1}$ 和 $e_{2}, \mu$ 是 $G \rtimes_{\sigma} K$ 上 的正规 2- 上循环, $\mathcal{M}$ 是作用在 Hilbert 空间 $\mathcal{H}$ 上的 von Neumann 代数, $\alpha$ 是群 $G \rtimes_{\sigma} K$ 在 $\mathcal{M}$ 上的自 同构表示, 且由酉表示 $U:(g, k) \mapsto U(g, k)$ 在 $\mathcal{H}$ 上实现, 则由定义 2.4 可以得到作用在 $\mathcal{H} \otimes l^{2}\left(G \rtimes_{\sigma} K\right)$ 上的 2- 上循环交叉积 $\mathcal{M} \rtimes_{\alpha}^{\mu}\left(G \rtimes_{\sigma} K\right)$.

由于群 $G$ 能够嵌入到半直积群 $G \rtimes_{\sigma} K$ 中, 因此群 $G$ 在 $\mathcal{M}$ 上的自同构表示 $\alpha^{\prime}$ 能够由西表示 $U^{\prime}: g \mapsto U\left(g, e_{2}\right)$ 在 $\mathcal{H}$ 上实现. 定义群 $G$ 上的正规 2- 上循环 $\mu^{\prime}$ 如下:

$$
\mu^{\prime}\left(g_{1}, g_{2}\right)=\mu\left(\left(g_{1}, e_{2}\right),\left(g_{2}, e_{2}\right)\right), \quad \forall g_{1}, g_{2} \in G,
$$

则根据定义 2.4 可以构造作用在 $\mathcal{H} \otimes l^{2}(G)$ 上的 2- 上循环交叉积 $\mathcal{M} \rtimes_{\alpha^{\prime}}^{\mu^{\prime}} G$.

$\forall k \in K$, 定义 Hilbert 空间 $l^{2}(G)$ 上的算子 $V(k)$ 如下:

$$
V(k)(\xi)(g)=\xi\left(\sigma\left(k^{-1}\right)(g)\right), \quad \forall \xi \in l^{2}(G), g \in G,
$$

容易验证 $V(k)$ 是 $l^{2}(G)$ 上的西算子. $\forall k \in K$, 令

$$
\beta(k)=\operatorname{Ad}\left(U\left(e_{1}, k\right) \otimes V(k)\right),
$$

其中

$\operatorname{Ad}\left(U\left(e_{1}, k\right) \otimes V(k)\right)(S \otimes T)=U\left(e_{1}, k\right) S U\left(e_{1}, k\right)^{*} \otimes V(k) T V(k)^{*}, \quad \forall S \in \mathcal{B}(\mathcal{H}), T \in \mathcal{B}\left(l^{2}(G)\right)$, 则 $\beta(k) \in \operatorname{Aut}\left(\mathcal{B}(\mathcal{H}) \bar{\otimes} \mathcal{B}\left(l^{2}(G)\right)\right)$ 且 $\beta: k \mapsto \beta(k)$ 是群 $K$ 在 $\mathcal{B}(\mathcal{H}) \bar{\otimes} \mathcal{B}\left(l^{2}(G)\right)$ 上的自同构表示.

引理 3.1 如果群 $G$ 上的限制的 2- 上循环 $\mu^{\prime}$ 满足

$$
\mu^{\prime}\left(g_{1}, g_{2}\right)=\mu^{\prime}\left(\sigma(k)\left(g_{1}\right), \sigma(k)\left(g_{2}\right)\right), \quad \forall g_{1}, g_{2} \in G, k \in K,
$$

则 (3.2) 中的 $\beta$ 是群 $K$ 在 2- 上循环交叉积 $\mathcal{M} \rtimes_{\alpha^{\prime}}^{\mu^{\prime}} G$ 上的自同构表示.

证明 $\forall k \in K, A U^{\prime}(g) \otimes L_{\mu^{\prime}}(g) \in \mathcal{M} \rtimes_{\alpha^{\prime}}^{\mu^{\prime}} G$, 由 (1.2) 和 (3.3) 可知,

$$
\begin{aligned}
V(k) L_{\mu^{\prime}}(g) V(k)^{*}(\xi)(h) & =L_{\mu^{\prime}}(g) V(k)^{*}(\xi)\left(\sigma\left(k^{-1}\right)(h)\right) \\
& =\mu^{\prime}\left(g, g^{-1} \sigma\left(k^{-1}\right)(h)\right) V(k)^{*}(\xi)\left(g^{-1} \sigma\left(k^{-1}\right)(h)\right) \\
& =\mu^{\prime}\left(g, g^{-1} \sigma\left(k^{-1}\right)(h)\right)(\xi)\left(\left(\sigma(k)\left(g^{-1}\right)\right) h\right) \\
& =\overline{\mu^{\prime}\left(\sigma(k)(g), \sigma(k)\left(g^{-1}\right) h\right)} \mu^{\prime}\left(g, g^{-1} \sigma\left(k^{-1}\right)(h)\right) L_{\mu^{\prime}}(\sigma(k)(g))(\xi)(h) \\
& =L_{\mu^{\prime}}(\sigma(k)(g))(\xi)(h) \quad\left(\forall \xi \in l^{2}(G), h \in G\right),
\end{aligned}
$$

即 $V(k) L_{\mu^{\prime}}(g) V(k)^{*}=L_{\mu^{\prime}}(\sigma(k)(g))$, 所以

$$
\begin{aligned}
\beta(k)\left(A U(g) \otimes L_{\mu^{\prime}}(g)\right) & =U\left(e_{1}, k\right) A U^{\prime}(g) U\left(e_{1}, k\right)^{*} \otimes V(k) L_{\mu^{\prime}}(g) V(k)^{*} \\
& =\left(U\left(e_{1}, k\right) A U\left(e_{1}, k\right)^{*}\right) U\left(e_{1}, k\right) U\left(g, e_{2}\right) U\left(e_{1}, k\right)^{*} \otimes V(k) L_{\mu^{\prime}}(g) V(k)^{*} \\
& =\left(U\left(e_{1}, k\right) A U\left(e_{1}, k\right)^{*}\right) U\left(\sigma(k)(g), e_{2}\right) \otimes V(k) L_{\mu^{\prime}}(g) V(k)^{*} \\
& =\alpha\left(e_{1}, k\right)(A) U\left(\sigma(k)(g), e_{2}\right) \otimes L_{\mu^{\prime}}(\sigma(k)(g)) .
\end{aligned}
$$


由此推出 $\beta(k)\left(\mathcal{M} \rtimes_{\alpha^{\prime}}^{\mu^{\prime}} G\right) \subseteq \mathcal{M} \rtimes_{\alpha^{\prime}}^{\mu^{\prime}} G$. 由于集合 $\left\{A U^{\prime}(g) \otimes L_{\mu^{\prime}}(g) \mid A \in \mathcal{M}, g \in G\right\}$ 的线性扩张在 2上循环交叉积 $\mathcal{M} \rtimes_{\alpha^{\prime}}^{\mu^{\prime}} G$ 中稠密且 $\beta(k) \in \operatorname{Aut}\left(\mathcal{B}(\mathcal{H}) \otimes \mathcal{B}\left(l^{2}(G)\right)\right)$, 所以上述 $\beta$ 是群 $K$ 在 2- 上循环交 叉积 $\mathcal{M} \rtimes_{\alpha^{\prime}}^{\mu^{\prime}} G$ 上的自同构表示.

由于群 $K$ 能够嵌入到半直积群 $G \rtimes_{\sigma} K$ 中, 所以可以定义群 $K$ 上的正规 2- 上循环 $\mu^{\prime \prime}$ 如下:

$$
\mu^{\prime \prime}\left(k_{1}, k_{2}\right)=\mu\left(\left(e_{1}, k_{1}\right),\left(e_{1}, k_{2}\right)\right), \quad \forall k_{1}, k_{2} \in K .
$$

从而, 根据定义 2.4, 我们可以构造作用在 $\mathcal{H} \otimes l^{2}(G) \otimes l^{2}(K)$ 上的 2- 上循环交叉积 $\left(\mathcal{M} \rtimes_{\alpha^{\prime}}^{\mu^{\prime}} G\right) \rtimes_{\beta}^{\mu^{\prime \prime}} K$. 现在, 我们证明 2- 上循环交叉积 $\mathcal{M} \rtimes_{\alpha}^{\mu}\left(G \rtimes_{\sigma} K\right)$ 同构于 2- 上循环交叉积 $\left(\mathcal{M} \rtimes_{\alpha^{\prime}}^{\mu^{\prime}} G\right) \rtimes_{\beta}^{\mu^{\prime \prime}} K$.

定理 3.2 设半直积群 $G \rtimes_{\sigma} K$ 上的正规 2- 上循环 $\mu$ 满足下列条件:

(i) 群 $G$ 上的限制 2- 上循环 $\mu^{\prime}$ 满足

$$
\mu^{\prime}\left(g_{1}, g_{2}\right)=\mu^{\prime}\left(\sigma(k)\left(g_{1}\right), \sigma(k)\left(g_{2}\right)\right), \quad \forall g_{1}, g_{2} \in G, k \in K
$$

(i) $\mu\left((g, k),(g, k)^{-1}(h, l)\right)=\mu^{\prime}\left(g, g^{-1} h\right) \mu^{\prime \prime}\left(k, k^{-1} l\right), \forall g, h \in G, k, l \in K$.

则 2- 上循环交叉积 $\mathcal{M} \rtimes_{\alpha}^{\mu}\left(G \rtimes_{\sigma} K\right) *$ 同构于 $\left(\mathcal{M} \rtimes_{\alpha^{\prime}}^{\mu^{\prime}} G\right) \rtimes_{\beta}^{\mu^{\prime \prime}} K$.

证明 定义从 Hilbert 空间 $l^{2}\left(G \rtimes_{\sigma} K\right)$ 到 Hilbert 空间 $l^{2}(G) \otimes l^{2}(K)$ 上的算子 $W$ 为

$$
W\left(\sum_{(h, l) \in G \rtimes_{\sigma} K} \xi(h, l) \chi_{(h, l)}\right)=\sum_{h \in G, l \in K} \xi(h, l) \chi_{h} \otimes \chi_{l},
$$

则 $W$ 是酉算子.

由于

$$
\begin{aligned}
W & L_{\mu}(g, k) W^{*}\left(\sum_{h \in G, l \in K} \xi(h, l) \chi_{h} \otimes \chi_{l}\right)=W L_{\mu}(g, k)\left(\sum_{(h, l) \in G \rtimes_{\sigma} K} \xi(h, l) \chi_{(h, l)}\right) \\
& =W\left(\sum_{(h, l) \in G \rtimes_{\sigma} K} \mu((g, k),(h, l)) \xi(h, l) \chi_{(g, k)(h, l)}\right) \\
& =W\left(\sum_{(h, l) \in G \rtimes_{\sigma} K} \mu\left((g, k),(g, k)^{-1}(h, l)\right) \xi\left((g, k)^{-1}(h, l)\right) \chi_{(h, l)}\right) \\
& =\sum_{h \in G, l \in K} \mu\left((g, k),(g, k)^{-1}(h, l)\right) \xi\left((g, k)^{-1}(h, l)\right) \chi_{h} \otimes \chi_{l},
\end{aligned}
$$

且

$$
\begin{aligned}
L_{\mu^{\prime}} & (g) V(k) \otimes L_{\mu^{\prime \prime}}(k)\left(\sum_{h \in G, l \in K} \xi(h, l) \chi_{h} \otimes \chi_{l}\right) \\
& =\sum_{h \in G, l \in K} \xi(h, l) L_{\mu^{\prime}}(g) V(k) \chi_{h} \otimes L_{\mu^{\prime \prime}}(k) \chi_{l} \\
& =\sum_{h \in G, l \in K} \xi(h, l) L_{\mu^{\prime}}(g) \chi_{\sigma(k) h} \otimes L_{\mu^{\prime \prime}}(k) \chi_{l} \\
& =\sum_{h \in G, l \in K} \xi(h, l) \mu^{\prime}(g, \sigma(k) h) \mu^{\prime \prime}(k, l) \chi_{g \sigma(k) h} \otimes \chi_{k l} \\
& =\sum_{h \in G, l \in K} \xi\left((g, k)^{-1}(h, l)\right) \mu^{\prime}\left(g, g^{-1} h\right) \mu^{\prime \prime}\left(k, k^{-1} l\right) \chi_{h} \otimes \chi_{l},
\end{aligned}
$$


则由条件 $(\dot{2})$ 可知,

$$
W L_{\mu}(g, k) W^{*}=L_{\mu^{\prime}}(g) V(k) \otimes L_{\mu^{\prime \prime}}(k) .
$$

容易验证

$$
A U^{\prime}(g) U\left(e_{1}, k\right)=A U(g, k), \quad \forall g \in G, k \in K, A \in \mathcal{M} .
$$

由于集合 $\left\{A U(g, k) \otimes L_{\mu}(g, k) \mid A \in \mathcal{M},(g, k) \in G \rtimes_{\sigma} K\right\}$ 的线性扩张在 2- 上循环交叉积 $\mathcal{M} \rtimes_{\alpha}^{\mu}\left(G \rtimes_{\sigma} K\right)$ 中稠密, 而集合 $\left\{A U^{\prime}(g) U^{\prime \prime}(k) \otimes L_{\mu^{\prime}}(g) V(k) \otimes L_{\mu^{\prime \prime}}(k) \mid A \in \mathcal{M}, g \in G, k \in K\right\}$ 的线性扩张在 2- 上循环 交叉积 $\left(\mathcal{M} \rtimes_{\alpha^{\prime}}^{\mu^{\prime}} G\right) \rtimes_{\beta}^{\mu^{\prime \prime}} K$ 中稠密, 所以由 (3.4) 和 (3.5) 可知, 2- 上循环交叉积 $\mathcal{M} \rtimes_{\alpha}^{\mu}\left(G \rtimes_{\sigma} K\right) *$ - 同 构于 $\left(\mathcal{M} \rtimes_{\alpha^{\prime}}^{\mu^{\prime}} G\right) \rtimes_{\beta}^{\mu^{\prime \prime}} K$.

$\forall g, h \in G, k, l \in K$, 取 $\mu((g, k),(h, l))=1$, 则 $\mu$ 是 $G \rtimes_{\sigma} K$ 上的正规 2- 上循环且满足定理 3.2 中 的条件 (i) 和 (2). 因此, 我们可以得到文献 [12] 中定理 3.3 .

注 3.3 ${ }^{[12]}$ 离散交叉积 $\mathcal{M} \rtimes_{\alpha}\left(G \rtimes_{\sigma} K\right) *$ - 同构于离散交叉积 $\left(\mathcal{M} \rtimes_{\alpha} G\right) \rtimes_{\beta} K$.

当 von Neumann 代数 $\mathcal{M}=\mathbb{C} I$ 时, $2-$ 上循环交叉积 $\mathcal{M} \rtimes_{\alpha}^{\mu} G$ 是 2 - 上循环群 von Neumann 代数 $\mathcal{L}_{\mu}(G)$. 因此, 我们得到下面的推论:

推论 3.4 设半直积群 $G \rtimes_{\sigma} K$ 上的正规 2- 上循环 $\mu$ 满足下列条件:

(i) 群 $G$ 上的限制 $2-$ 上循环 $\mu^{\prime}$ 满足

$$
\mu^{\prime}\left(g_{1}, g_{2}\right)=\mu^{\prime}\left(\sigma(k)\left(g_{1}\right), \sigma(k)\left(g_{2}\right)\right), \quad \forall g_{1}, g_{2} \in G, k \in K ;
$$

$(\dot{2}) \mu\left((g, k),(g, k)^{-1}(h, l)\right)=\mu^{\prime}\left(g, g^{-1} h\right) \mu^{\prime \prime}\left(k, k^{-1} l\right), \forall g, h \in G, k, l \in K$.

则 2- 上循环群 von Neumann 代数 $\mathcal{L}_{\mu}\left(G \rtimes_{\sigma} K\right) *$ - 同构于 $\mathcal{L}_{\mu^{\prime}}(G) \rtimes_{\beta}^{\mu^{\prime \prime}} K$.

下面将举例说明满足定理 3.2 中条件 (i) 和 $(\dot{2})$ 的非平凡的 $2-$ 上循环 $\mu$ 是存在的.

例 3.5 令 $G$ 是加法群 $\mathbb{Z}^{2}, K$ 为特殊线性群 $S L(2, \mathbb{Z}) . \forall B \in S L(2, \mathbb{Z})$, 定义群 $\mathbb{Z}^{2}$ 上的映射 $\sigma_{B}$ 为 $\sigma_{B}(x)=B x$, 则 $\sigma_{B}$ 是群 $\mathbb{Z}^{2}$ 上的自同构, 而 $\sigma: B \mapsto \sigma_{B}$ 是群 $S L(2, \mathbb{Z})$ 到 $\operatorname{Aut}\left(\mathbb{Z}^{2}\right)$ 中的同态. 利用 群 $\mathbb{Z}^{2}$ 和 $S L(2, \mathbb{Z})$ 构造半直积群 $\mathbb{Z}^{2} \rtimes_{\sigma} S L(2, \mathbb{Z})$. 对于数 $\lambda \in \mathbb{T}$, 定义

$$
\mu((x, A),(y, B))=\lambda^{\frac{1}{2}|x, A y|},
$$

其中 $|x, A y|$ 表示矩阵 $(x, A y)_{2 \times 2}$ 的行列式. 由于 $\forall x, y, z \in \mathbb{Z}^{2}, A, B, C \in S L(2, \mathbb{Z})$,

$$
\mu((0, E),(x, A))=\lambda^{\frac{1}{2}|0, x|}=1=\mu((x, A),(0, E)),
$$

且

$$
\begin{aligned}
\mu & ((x, A),(y, B)) \mu((x, A)(y, B),(z, C)) \\
& =\mu((x, A),(y, B)) \mu((x+A y, A B),(z, C)) \\
& =\lambda^{\frac{1}{2}|x, A y|} \lambda^{\frac{1}{2}|x+A y, A B z|}=\lambda^{\frac{1}{2}(|x, A y|+|x+A y, A B z|)} \\
& =\lambda^{\frac{1}{2}(|x, A y|+|x, A B z|+|A y, A B z|)}=\lambda^{\frac{1}{2}(|x, A y|+|x, A B z|+|y, B z|)} \\
& =\lambda^{\frac{1}{2}(|x, A y+A B z|+|y, B z|)}=\lambda^{\frac{1}{2}|x, A y+A B z|} \lambda^{\frac{1}{2}|y, B z|} \\
& =\mu((x, A),(y+B z, B C)) \mu((y, B),(z, C)) \\
& =\mu((y, B),(z, C)) \mu((x, A),(y, B)(z, C)),
\end{aligned}
$$

所以 $\mu$ 是群 $\mathbb{Z}^{2} \rtimes_{\sigma} S L(2, \mathbb{Z})$ 上的正规 2- 上循环. 
(i) 由于

$$
\mu^{\prime}(x, y)=\mu((x, E),(y, E))=\lambda^{\frac{1}{2}|x, y|}
$$

且

$$
\mu^{\prime}(\sigma(A)(x), \sigma(A)(y))=\lambda^{\frac{1}{2}|A x, A y|}=\lambda^{\frac{1}{2}|x, y|}=\mu^{\prime}(x, y),
$$

所以定理 3.2 中的条件 (i) 成立.

(2) 由于

$$
\begin{aligned}
\mu\left((x, A),(x, A)^{-1}(y, B)\right) & =\mu\left((x, A),\left(\sigma\left(A^{-1}\right)(y-x), A^{-1} B\right)\right) \\
& =\mu\left((x, A),\left(A^{-1}(y-x), A^{-1} B\right)\right) \\
& =\lambda^{\frac{1}{2}|x, y-x|}=\lambda^{\frac{1}{2}|x, y|}
\end{aligned}
$$

且

$$
\mu^{\prime}(x, y-x) \mu^{\prime \prime}\left(A, A^{-1} B\right)=\lambda^{\frac{1}{2}|x, y-x|} \mu\left((0, A),\left(0, A^{-1} B\right)\right)=\lambda^{\frac{1}{2}|x, y|},
$$

所以定理 3.2 中的条件 $(\dot{2})$ 成立.

\section{参考文献}

1 Murray F J, von Neumann J. On rings of operators. Ann Math, 1936, 37: 116-229

2 Murray F J, von Neumann J. On rings of operators IV. Ann Math, 1943, 44: 716-808

3 Voiculescu D, Dykema K, Nica A. Free Random Variable. CRM Monogr Ser, vol. 1. Providence, RI: Amer Math Soc, 1992

4 Popa S. On Ozawa's property for free group factors. Int Math Res Notices, 2007, doi:10.1093/imrn/rnm036

5 Vaes S. An inner amenable group whose von Neumann algebra does not have property Gamma. arXiv: 0909.1485, 2009

6 Ioana A, Popa S, Vaes S. A class of superrigid group von Neumann algebras. arXiv: 1007.1412v1, 2010

7 Connes A, Jones V F R. Property (T) for von Neumann algebras. Bull London Math Soc, 1985, 17: 57-62

8 Nicoara R, Popa S, Sasyk R. On $I I_{1}$ factors arising from 2-cocycles of $w$-rigid groups. J Funct Anal, 2007, 242: 230-246

9 Turumaru T. Crossed products of operator algebras. Tohoku Math J, 1958, 10: 335-365

10 Nakamura M, Takeda Z. On some elementary properties of the crossed product of von Neumann algebras. Proc Japan Acad Ser A Math Sci, 1958, 34: 489-494

11 Kadison R V, Ringrose J. Fundamentals of the Theory of Operator Algebras, vols. I and II. Orlando: Academic Press, 1983 and 1986

12 吴文明, 袁巍. von Neumann 代数交叉积的注记. 数学学报, 2008, 51: 803-808

13 吴文明. 与群 $P S L_{2}(\mathcal{R})$ 相关的交叉积 $\mathcal{R}(\mathcal{A}, \alpha)$ 的一点注记. 中国科学 $\mathrm{A}$ 辑, 2007, 37: 1283-1290

14 张小霞, 郭㯟正. Woronowicz $\mathrm{C}^{*}$ 代数交叉积的正则与协变表示. 中国科学 $\mathrm{A}$ 辑, 2005, 35: 982-996

\section{2-Cocycle crossed products}

\section{ZHAO JianWei}

Abstract A 2-cocycle crossed product is defined in this paper given a von Neumann algebra $\mathcal{M}$ and a discrete group $G$ with a normalized 2-cocycle $\mu$ on it, which generalizes the classical discrete crossed product. Also, the associative law of the 2-cocycle crossed product is shown in this paper.

Keywords: von Neumann algebra, 2-cocycle crossed product, associative law

MSC(2000): 46L10

doi: $\quad 10.1360 / 012010-762$ 